\title{
Sciendo
}

RURAL SUSTAINABILITY RESEARCH 43(338), 2020

ISSN - 2256-0939

(c) Latvia University of Life Sciences and Technologies, all rights reserved http://www.llu.Iv/en/

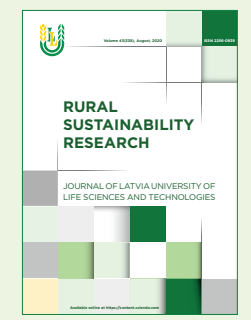

Received: 22 April 2020

Revised: 15 June 2020

Accepted: 10 July 2020

\section{Civil Engineering Inspection for Real Estate Evaluation with the Use of Artificial Learning Algorithms and Fuzzy Logic}

\author{
*Vladimir Surgelas ${ }^{1}$, Irina Arhipova ${ }^{2}$, Vivita Pukite ${ }^{1}$ \\ ${ }^{1}$ Faculty of Environment and Civil Engineering, Latvia University of Life Sciences and Technologies, \\ ${ }^{2}$ Faculty of Information Technologies, Latvia University of Life Sciences and Technologies, \\ Jelgava, Latvia \\ ORCID ID: *Vladimir Surgelas https://orcid.org/0000-0002-8317-6225 \\ Irina Arhipova https://orcid.org/0000-0003-1036-2024 \\ Vivita Pukite https://orcid.org/0000-0002-0529-8200
}

\begin{abstract}
The technical inspection of a building carried out by an expert in civil engineering can identify and classify the physical conditions of the real estate; this generates relevant information for the protection and safety of users. Given the real conditions of the property, and for the real estate valuation universe, using artificial intelligence and fuzzy logic, it is possible to obtain the market price associated with the physical conditions of the building. The objective of this experiment is to develop a property evaluation model using a civil engineering inspection form associated with artificial intelligence, and fuzzy logic, and also compare with market value to verify the applicability of this inspection form. Therefore, the methodology used is based on technical inspection of civil engineering regarding the state of conservation of properties according to the model used in Portugal and adapted to the reality of Latvia. Artificial intelligence is applied after obtaining data from that report. From this, association rules are obtained, which are used in the diffuse logic to obtain the price of the apartment per square meter, and for comparison with the market value. For this purpose, 48 samples of residential apartments located in the city of Jelgava in Latvia are used, with an inspection carried out from October to December 2019. The main result is the $9 \%$ error metric, which demonstrates the possibility of applying the method proposed in this experiment. Thus, for each apartment sample consulted, it resulted in the state of conservation and a market value associated.
\end{abstract}

Keywords: Real estate appraisal, Fuzzy logic, data mining, civil engineering.

\section{Introduction}

Civil engineering, focused on real estate appraisal, is a specialty that covers a wide range of knowledge in the areas of civil engineering and architecture, in addition to other related fields. A property valuation focuses on determining the market value of properties. The amount to be estimated is usually based on several factors.

In this case, it is important to observe the relationship between these selected variables to verify the dependencies that might be or might not be between them (Darlington et al., 2019). Thus, for the correct specification of the dependent variable, a market investigation is necessary regarding its conduct and the forms of expression of the price (such as total or unit price, reference currency, payment methods) (Butler, 1982), as well as observe the homogeneity in the measurement units (Lima, 2013). The independent variables refer to physical, location, and economic characteristics (for example, area, front, construction pattern, fiscal indices, distance to a pole of influence, offer/transaction, time, and business condition: spot or forward), among others. Independent variables can be divided basically into quantitative and qualitative variables (Del Giudice, De Paola, \& Cantisani, 2017).

However, the least-squares method cannot distinguish between direct and indirect effects of

\footnotetext{
* Corresponding Author's email: 
variables. From this, it is more reasonable to identify which variable (among those that have the same information in practice) is the most important and/or the most representative. A simple corrective measure for multicollinearity would be to increase the sample size. However, this procedure is not always the best option. Besides, there is also the occurrence of a limited number of samples available on the market, or when the study market has samples that are terribly like each other or interrelated (Butler, 1982).

To mitigate this situation, the diffuse model (Zadeh, 1999) can better adapt to some cases of real estate valuation in which these situations of redundancy occur (Kusan, Aytekin, \& Özdemir, 2010). In the cloudy model, there is greater flexibility in the application of analysis and reflection parameters that mimic human behavior. This can help decisionmakers as to which procedure is best to follow. In addition to the nebulous methodology, it contemplates the subjectivity of human thought (Brandalise, Pereira, \& Mello, 2019), a fact that could facilitate the application of the method without losing the scientific seal of the evaluation work. Fuzzy logic is a good technique for transforming belief variables into appropriate concepts for dealing with heuristic models that use linguistic terms. These linguistic terms are more understandable to people than mathematical models. Therefore, the heuristic process can lead to a quick assessment of the risks involved and, thus, also bring quick decisions (Veiga, 1994).

Currently, the application of fuzzy logic coupled with the formulation of association rules outlined by artificial intelligence can result in a more objective assessment process, in line with human thought, accordingly Weka Software. The Weka software is open source and provides practical tools for analyzing data and providing forecasts (Eibe, Mark, \& Ian, 2016). In the evaluation of properties, the descriptive characteristics of a specific property serve to estimate its final value, through logical propositions or linguistic rules. In this context, according to (Posselt, Frozza \& Molz, 2015) in Fuzzy Software is used as the analytical tool for this experiment. The Mamdani structure fits better with the nonlinear behavior of the real estate market. Thus, once a fuzzy set is obtained through the inference process, defuzzification is performed. In fuzzy logic, there are not only extreme answers, it allows us to assign a degree of approximation to the solution, seeking not only the exact answer but the proximity or not of that answer (Karaboga \& Kaya, 2019).

From this, this experiment is related to the property evaluation process. Thus, the possibility of using fuzzy strategies it mimics human behavior. This is based on rules of association and experience of specialists, rather than a control restricted only by mathematical models. Therefore, the diffuse model behaves according to the reasoning that the specialist uses to infer the rules of the association, based on information that he already knows or on dispersed data that have not yet become knowledge. Based on this principle and considering that the knowledge of the state of conservation of the building is the basis for making decisions about the conservation and rehabilitation measures to be implemented, it was considered appropriate to carry out a practical study on residential buildings. Thus, the objective of this experiment is to develop a property evaluation model using a civil engineering inspection form associated with artificial intelligence and fuzzy logic, in addition to comparing it with the market value to verify the applicability of this inspection form. This experiment considered 48 residential apartments, located in the city of Jelgava, in Latvia. The survey took place from September to December 2019.

\section{Materials and Methods}

The inspection criterion was inspired by a technique used in Portugal being adapted to the reality of Latvia to propose improvements in the evaluation process. These inspections of civil engineering coupled with the technique of fuzzy logic with the aid of artificial intelligence, expeditiously, allowed to determine the degree of deterioration of the apartment by identifying and characterizing its main anomalies occurred during the useful life of the property. This evaluation is made through a visual inspection, which detects the main anomalies present in the building's constituent elements. Through this proposed methodology, the properties are analyzed and, known their state of conservation and the existence of basic infrastructure can check the price forecast. In this sense, the proposed case aims to see the problem in a heuristic way, so that the anomalies of the buildings are classified into 5 basic categories: 1-Exceptionally light: No anomalies were detected or without importance. 2-Light: Anomalies that do not affect safety and detract from the appearance and require easy work to perform it; 3-Medium: Anomalies that do no affect safety; that detract from the appearance and require hard work. Anomalies that detract from use and comfort and require work of repair, replacement, or easy-to-carry repair it. 4-Serious: Anomalies that affect safety but do not require urgent repair; Anomalies that hinder use and comfort and require difficult work. Anomalies that put health and/or safety at risk and can motivate accidents without gravity and request work of easy execution. 5- Profoundly serious: Anomalies that affect safety and require urgent repair. Anomalies that endanger the health and/or safety and can motivate 
accidents without seriousness and requesting work of easy execution. Anomalies that put at risk and or safety and can motivate serious accidents, absence, or inoperability of basic infrastructure.

So, for a better understanding of the methodology used in this experiment, it was divided into 4 Steps. Step 1

For this experiment, 48 samples were selected randomly from 1 to 3 bedroom apartments located in the center of Jelgava in Latvia. These samples of apartments advertised for sale range from 23 square meters to 84 square meters. To carry out the identification, characterization, and classification of these properties, we used the technical form of the Method of Evaluation of the State of Conservation of Buildings of the Ministry of Public Works, Transport and Communications of Portugal (MAEC, 2006), this in a way adapted to the Latvian reality, and so does not use the description of the details of the pathologies, as they are supplements that do not interfere in this experiment for Latvia. The MAEC's form contains 37 items, applied in the experiment, and covers the internal and external conditions of the building, from the structure to fire fighting facilities, among others. This for qualitative analysis of anomalies of functional elements of the building and the apartment.

The technical form is recommended for application according to the expertise of the civil engineer. The qualitative analysis considers indices for each anomaly found. The variables of these anomalies range from very severe to severe, in a total of 5 levels, except for the item not applicable. From the anomalies identified in each functional element, the scores are counted. The calculation of scores considers the product between the number of associated points at each anomaly level and the weight given to the functional element. The weighting assigned uses a parameter from 1 to 6, according to MAEC (2006). This parameter was suggested and verified by technicians from the National Civil Engineering Laboratory of Portugal (LNEC). In this experiment in Latvia, the same considerations and weightings are used to obtain the referred scores, where the property's anomaly index is obtained. The calculation of this index is a sum of the scores divided by the sum of the weights. From there, to obtain the anomaly index of each building, an anomaly scale is used as mentioned in MAEC (2006) (Table 1).

\section{Step 2}

In this step, from the data obtained in Step 1, the use of artificial intelligence to obtain association rules begins. For that, data mining is necessary. For this task, the Apriori algorithm was chosen. Apriori performs the mining of association rules. This algorithm is suitable for a small amount of data, ideal for this experiment. The purpose of the Apriori algorithm is to generate association rules based on the number of times that two or more items occur together in the set of transactions; it performs various combinations between items.

The author prepares the specific file from the data and attributes collected in the civil engineering inspection. Of the experiment's attributes, the price per square meter, the area of each apartment, the apartment's conservation index, in the case of new, renovated, or old ones, the building's conservation index, whether new, renovated or old, are used. The data is organized by the author to aggregate the attributes of each apartment, which are inserted in the Weka software to generate association rules used in Fuzzy Logic.

The application of the .arff file developed by the author is later launched in the Weka software. So, the file to Weka software was successfully built being aided by the text editor computational tool. This Weka will generate association rules that will present a pattern of behavior, which in this case are related to the data collected from the real estate samples. The Weka software is open source and provides practical tools for analyzing data and providing forecasts, to perform modern probabilistic modeling. After that, the next step is to perform the fuzzy modeling. For this, the computational tool, InFuzzy software, is used.

Step 3

In this step, the InFuzzy software is used, which is a tool for the development of diffuse system applications. In this task, the author inserts the association rules of interest chosen for this experiment, as selected in Step 2. The entrance variables: conservation index of the apartment, the area in a square meter, and conservation index of the building. The output variable is the price per square meter. The Fuzzy Inference process is fuzzification, rules, and defuzzification of the output by the center of gravity method. The methodology is based on fuzzy logic according to the following steps (Figure 1):

Table 1

Anomaly index (AI) concerning the building's conservation status, according to MAEC (2006)

\begin{tabular}{|c|c|c|c|c|}
\hline Exceptionally light & Light & Medium & Serious & Profoundly serious \\
\hline $5.00 \geq \mathrm{AI} \geq 4.50$ & $4.50>\mathrm{AI} \geq 3.50$ & $3.50>\mathrm{AI} \geq 2.50$ & $2.50>\mathrm{AI} \geq 1.50$ & $1.50>\mathrm{AI} \geq 1.00$ \\
\hline
\end{tabular}




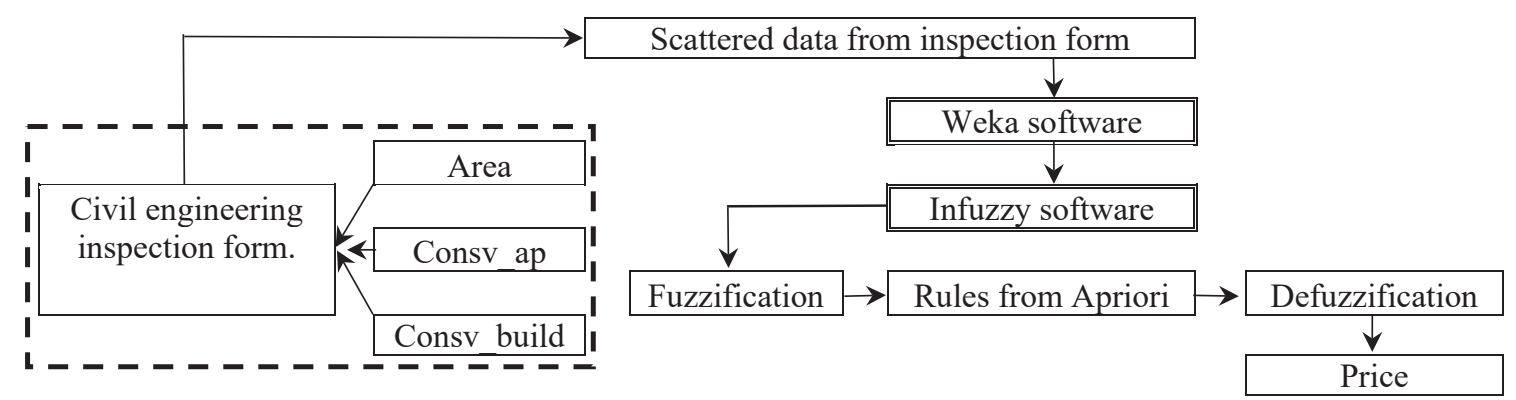

Source: created by the authors.

Figure 1. Boundaries of the experiment with artificial intelligence and fuzzy logic.

1-Inputs (variables - area, conservation apartment (Consv_ap), conservation build (Consv-build)); 2-Fuzzification of inputs (fuzzy weights); 3-Rules (from Apriori algorithm); 4-Defuzzification (sets the numerical value of the output $=$ price) .

InFuzzy software is a graphical interface tool, with a code for modeling diffuse system applications, with simulation capabilities. From that, to forecast the price of the property, the Infuzzy software is used to model the diffuse system, where it is necessary to create a tree model, using linguistic terms to generate a function per variable (Table 4), which are established by the author. The input variables used are those indexes obtained through the civil engineering inspection form, which is: apartment conservation index, building conservation index, room, area, used for fuzzification.

As for the Fuzzy classification parameters, in the case of the area variable, the Chauvenet criterion is used to obtain the values of the association function (Table 4) whose values obtained can be used to determine the parameters of a Gaussian function. For this, the procedure follows as described below.

a. Initially selected 48 samples of 1, 2, 3 room apartments in Jelgava, Latvia, October to December 2019.

b. Calculated the mean (and standard deviation () of the sample roll.

c. Checked the relevance of the sample roll by Chauvenet's exclusion criterion. By this criterion, the sample is relevant if the quotient between its deviation (which is the difference in absolute value between the sample value and the mean) and the standard deviation is lower than the tabulated Chauvenet critical number.

d. Applied statistical theory: where = percentile values for the distribution " $t$ " Student to " $n$ " samples and " $n-1$ " degrees of freedom with the confidence of $80 \%$. The confidence limit is defined by the model:

$X_{\min }=\bar{x} \pm t_{c} \times \frac{s}{\sqrt{n-1}}$
Other parameters of the other two variables and their classifiers were obtained from Table 1.

The next task is to insert data from Step 1, and the association rules chosen by the author from those generated in Step 2 (Table 3). After that, proceed with a defuzzification. This is where the software makes a simulation where the prediction of the prices of the properties of the experiment is obtained.

Step 4

The objective of this step is to interpret the precision of the experimental model, and for that, it uses error metrics to evaluate predictions. The task here is to compare the price offered with the results obtained in step 3. For accuracy of the results the metrics used are the Root Mean Square Error (RMSE), Mean Absolute Error (MAE), and Mean Percentage Absolute Error (MAPE) respectively. The RMSE penalizes far more values that are far from the average. In the MAE it depends on scale, it is the average of the absolute difference between the realized and the predicted, and therefore if some values are extremely far from the average this is not relevant. In the case of MAPE, it evaluates the model with identification of losses in the regression problems, as it interprets the relative error intuitively, as it does not depend on a percentage scale, here this metric calculates the absolute difference between two parameters of this experiment, the result is given in terms of percentage evaluation metrics, according to the formula below. Evaluate the price forecasting accuracy by (RMSE), (MAE) and (MAPE).

$$
M A P E=\frac{1}{n} \sum_{t=1}^{n}\left(\frac{Y_{t}-\bar{\Upsilon}_{t}}{Y_{t}}\right)^{2} \times 100
$$

\section{Results and Discussion}

Step 1

From the samples of the 48 apartments advertised for sale, 37 items are analyzed from civil engineering, resulting in obtaining the indexes of the state of conservation of these properties. These involve the internal aspect of the apartment and the external 
Anomalies obtained from the civil engineering inspection form

\begin{tabular}{|l|c|c|c|c|c|}
\hline \multirow{2}{*}{ Variables } & \multicolumn{4}{|c|}{ Amount of anomalies found after civil engineering inspection } \\
\cline { 2 - 6 } & $\begin{array}{c}\text { Exceptionally } \\
\text { light }\end{array}$ & Light & Medium & Serious & $\begin{array}{c}\text { Profoundly } \\
\text { serious }\end{array}$ \\
\hline Consv_ap & 03 & 22 & 17 & 03 & 03 \\
\hline Consv_buid & 00 & 00 & 34 & 10 & 04 \\
\hline
\end{tabular}

aspect of the building. Of the results obtained in the apartments, referring to consv_ap (Table 2), there are approximately $46 \%$ in good condition, which means, in summary, that little maintenance expenses are necessary. Apartments that need some type of maintenance whose anomaly needs repairs that are difficult to use and that require a lot of work are approximately $35 \%$, while the bad (serious) and extremely bad index is approximately $12 \%$, and approximately $7 \%$ are in profoundly serious condition generating unexpected risks due to the lack of security of the facilities, among other important factors. This means that property prices must vary due to the state of conservation of these properties.

For the case of external conservation of the building, consulting the variable consv_bulid (Table 2), everyone needs repairs. About $71 \%$ are classified as medium. Thus, those who need repairs that impair their appearance are soon less attractive to buy, which can take a long time to be transacted, as they generate huge expenses. Of the buildings with a bad and serious and profoundly serious index, they are approximately $29 \%$ reflecting on bad visual pollution and bad architecture among other factors, and that need urgent repairs. Therefore, corrective actions are essential in all inspected buildings. With the detailed obtaining of the properties, referring to the areas consv_apar and consv_build, the anomaly indices are specified in table 05 as an input (variable). The other data for the continuation of the experiment were organized in the .arff file in the subsequent step.
Step 2

To insert the file format necessary for the Weka software to understand, which is not obtained automatically, the domain of the attribute is described by the author. The author created the arff file on the Notepad with the content of the price /square meter ratio; the attributes of the experiment used are the price per square meter obtained for each property; area of each property in square meters; the index obtained from the inspection sheet for the variables: consv ap; and consv_build; the variable number of rooms; and the price variable is defined with the following linguistic definition: low, medium and expensive. With the domain of the described attribute, the .arff file was generated. After that, the file .arff is loaded into Weka to obtain the mining rules using the Apriori algorithm. Thus, by the WEKA software, 49 association rules were generated by the data mining/learning algorithm. The author chooses all the rules that contain price as a consequence, which are 7 (Table 3). After analyzing the purpose of price prediction in this first result, 7 best rules following $60 \%$ (confidence) were chosen. Step 3 starts with the association rules obtained here. Step 3

In this stage, the price of the property is forecast, using the Infuzzy software for modeling the diffuse system. The modeling of a tree is done using linguistic terms to generate a function per variable.

In the simulation of the experiment, the input variables defined in the tree are configured by the author to define the experiment. In this way, the

Table 3

Association rules are chosen by the author, from the Weka software

\begin{tabular}{|c|c|l|l|l|}
\hline 1 & IF & $($ consv_ap $=$ good $)$ AND $($ consv_buid = rebew $)$ & THEN & $($ Price $=$ expensive $)$ \\
\hline 2 & IF & $($ consv_ap $=$ medium $)$ & THEN & $($ Price $=$ average $)$ \\
\hline 3 & IF & $($ consv_ap $=$ medium $)$ AND $($ consv_build $=$ old $)$ & THEN & $($ Price $=$ average $)$ \\
\hline 4 & IF & $($ consv_build $=$ renew $)$ & THEN & $($ Price $=$ expensive $)$ \\
\hline 5 & IF & $($ consv_ap $=$ good $)$ & THEN & $($ Price $=$ expensive $)$ \\
\hline 6 & IF & $($ consv_ap $=$ good $)$ AND $($ consv_build $=$ old $)$ & THEN & $($ Price $=$ expensive $)$ \\
\hline 7 & IF & $($ consv_ap $=$ old $)$ & THEN & (Price $=$ average $)$ \\
\hline
\end{tabular}


variables, scope, classification, parameter, and association function are defined (Table 4). To define the parameter item in the area variable, the author chooses a Gaussian function, defined below.

From the choice of the best association rules, seven (7) association rules (Table 3 ) are informed in the infuzzy software. After that, the author performs the simulation of this experiment in the InFuzzy software. The result obtained is the price forecast (Table 5) of each property. Therefore, the price of the property is based on the degree of anomalies identified from the civil engineering inspection form. Note, the Time (ms) result is the processing time for each simulation.

The average price for standard residential apartments in July in Jelgava was 522 EUR m$^{-2}$. This information is an overview of the apartment market (http://liaa.gov.lv/en/business-latvia/real-estatemarket-research). However, the website www.liaa. gov.lv does not report the conservation status of the residential properties traded. On the other hand, in the task of defuzzification (Figure 2), considering that most of the residential apartments in Jelgava are in urgent need of renovation, these values (Table 2) of the fuzzy logic are very good references if you compare with the reality in the city of Jelgava, therefore, the suggested decompression value is approximately $416 \mathrm{EUR} \mathrm{m}^{-2}$.

The predicted values in the fuzzy model for each apartment in the sample are derived from the different conservation indices as a function of civil engineering inspection. This was possible due to the proper compilation of scattered data found in the market, which had not been properly processed before. However, if this scattered data is used correctly, it can become knowledge. Thus, the defuzzification process shows the output variable (price at 416.67 EUR), this value is per square meter (Figure 2).

By the analysis of the traditional statistical process, where:

Calculation of sample average

$$
\left(\quad=48.58 \mathrm{~m}^{-2}\right.
$$

The standard deviation of the sample.

() $=13.44$

Percentile values for Student's t-distribution.

$(t c)=2.556$ (interpolated table value)

Sample numbers

$(n) \quad=48$

Degrees of freedom

$(n-1)=47$

Confidence limits were defined by the Chavenaut criterion, according to the following model.

$$
X_{\min }=\bar{x} \pm t_{c} \times \frac{S}{\sqrt{n-1}} \rightarrow\left\{\begin{array}{l}
\mathrm{X}_{\max }=53,64 \mathrm{~m}^{-2} \\
\mathrm{X}_{\min }=43,51 \mathrm{~m}^{-2}
\end{array}\right.
$$

Table 4

\begin{tabular}{|c|c|c|c|c|c|c|c|}
\hline & Variables & Range & Classification & \multicolumn{3}{|c|}{ Parameters } & Pertinency function \\
\hline \multirow{11}{*}{ 芌 } & \multirow{3}{*}{ AREA } & \multirow{3}{*}[0,100]{} & low & 0.00 & & 43,51 & Left ramp \\
\hline & & & normal & 48.58 & & 13.44 & Gaussian \\
\hline & & & big & 53,64 & & 100 & Right ramp \\
\hline & \multirow{5}{*}{ CONSV_AP } & \multirow{5}{*}[0,5]{} & terrible & \multicolumn{3}{|c|}{0.0} & Left ramp \\
\hline & & & bad & \multicolumn{3}{|c|}{$\begin{array}{lll}1.5 & 2.0 & 2.5 \\
\end{array}$} & Triangle \\
\hline & & & medium & \multicolumn{3}{|c|}{$\begin{array}{lll}2.5 & 3.0 & 3.5\end{array}$} & Triangle \\
\hline & & & good & \multicolumn{3}{|c|}{$3.5 \quad 4.0$} & Trapezoidal \\
\hline & & & excellent & \multicolumn{3}{|c|}{$4.5 \quad 5.0$} & Right ramp \\
\hline & \multirow{3}{*}{ CONSV_BUILD } & \multirow{3}{*}[0,3]{} & new & & & 1 & Discreet \\
\hline & & & renew & & & 2 & Discreet \\
\hline & & & old & & & 3 & Discreet \\
\hline \multirow{3}{*}{ 芌 } & \multirow{3}{*}{$\Longrightarrow$ PRICE } & \multirow{3}{*}[0,1200]{} & low & & 150 & 300 & Left ramp \\
\hline & & & average & 250 & 400 & 600 & Triangle \\
\hline & & & expensive & & 550 & 600 & Right ramp \\
\hline
\end{tabular}

Fuzzy sets - Classifiers according to Infuzzy software 
Simulation from InFuzzy software - Output in EUR $\mathbf{m}^{-2}$

Table 5

\begin{tabular}{|c|c|c|c|c|}
\hline \multirow{2}{*}{$\begin{array}{l}\text { Time } \\
(\mathrm{ms})\end{array}$} & \multicolumn{3}{|c|}{ Input (variable) } & \multirow{2}{*}{$\begin{array}{c}\text { Output } \\
\text { (Price) } \\
\text { EUR }\end{array}$} \\
\hline & $\begin{array}{l}\text { Index: } \\
\text { consv_ap }\end{array}$ & $\begin{array}{l}\text { Area } \\
\left(\mathrm{m}^{2}\right)\end{array}$ & $\begin{array}{c}\text { Index: } \\
\text { consv_build }\end{array}$ & \\
\hline 63 & 3.20 & 48.00 & 3.00 & 416.67 \\
\hline 46 & 3.10 & 38.00 & 1.00 & 417.22 \\
\hline 46 & 2.40 & 44.00 & 3.00 & 416.67 \\
\hline 30 & 2.70 & 30.00 & 3.00 & 416.67 \\
\hline 46 & 1.20 & 23.00 & 3.00 & 416.67 \\
\hline 31 & 2.70 & 46.10 & 3.00 & 416.67 \\
\hline 46 & 4.30 & 62.00 & 3.00 & 693.26 \\
\hline 31 & 1.40 & 52.00 & 3.00 & 416,67 \\
\hline 46 & 3.80 & 43.00 & 2.00 & 887.31 \\
\hline 46 & 3.20 & 52.40 & 3.00 & 416.67 \\
\hline 47 & 3.61 & 42.00 & 3.00 & 623.87 \\
\hline 46 & 3.65 & 33.00 & 3.00 & 660.05 \\
\hline 46 & 3.70 & 40.30 & 2.00 & 887.31 \\
\hline 46 & 3.10 & 48.00 & 3.00 & 416.67 \\
\hline 46 & 1.30 & 55.40 & 3.00 & 416.67 \\
\hline 46 & 2.60 & 56.00 & 3.00 & 416.67 \\
\hline 46 & 1.70 & 36.00 & 3.00 & 416.67 \\
\hline 46 & 3.30 & 48.30 & 3.00 & 416.67 \\
\hline 46 & 4.70 & 61.00 & 2.00 & 887.31 \\
\hline 47 & 4.30 & 83.30 & 3.00 & 693.26 \\
\hline 31 & 4.31 & 56.00 & 3.00 & 687.42 \\
\hline 46 & 4.34 & 58.00 & 3.00 & 667.58 \\
\hline 46 & 3.20 & 40.40 & 3.00 & 416.67 \\
\hline 46 & 1.80 & 54.00 & 3.00 & 416.67 \\
\hline
\end{tabular}

\begin{tabular}{|c|c|c|c|c|}
\hline \multirow{2}{*}{$\begin{array}{l}\text { Time } \\
(\mathrm{ms})\end{array}$} & \multicolumn{3}{|c|}{ Input (variable) } & \multirow{2}{*}{$\begin{array}{c}\text { Output } \\
\text { (Price) } \\
\text { EUR }\end{array}$} \\
\hline & $\begin{array}{l}\text { Index: } \\
\text { consv_ap }\end{array}$ & $\begin{array}{l}\text { Area } \\
\left(\mathrm{m}^{2}\right)\end{array}$ & $\begin{array}{c}\text { Index: } \\
\text { consv_build }\end{array}$ & \\
\hline 46 & 4.80 & 41.00 & 3.00 & 416.67 \\
\hline 46 & 2.80 & 46.00 & 3.00 & 416.67 \\
\hline 31 & 3.20 & 30.00 & 3.00 & 416.67 \\
\hline 46 & 2.58 & 40.00 & 3.00 & 416.67 \\
\hline 47 & 4.20 & 84.00 & 3.00 & 737.32 \\
\hline 47 & 3.90 & 48.00 & 2.00 & 887.31 \\
\hline 46 & 2.60 & 69.00 & 3.00 & 416.67 \\
\hline 31 & 2.52 & 31.00 & 3.00 & 416.67 \\
\hline 46 & 3.60 & 42.18 & 2.00 & 887.31 \\
\hline 46 & 3.68 & 58.00 & 2.00 & 887.31 \\
\hline 45 & 3.30 & 58.00 & 2.00 & 818.03 \\
\hline 46 & 3.87 & 41.00 & 2.00 & 887.31 \\
\hline 47 & 4.30 & 68.70 & 2.00 & 887.31 \\
\hline 46 & 4.40 & 30.00 & 2.00 & 887.31 \\
\hline 47 & 4.50 & 63.00 & 1.00 & 600.00 \\
\hline 47 & 4.30 & 54.00 & 1.00 & 879.95 \\
\hline 46 & 4.20 & 66.00 & 1.00 & 882.42 \\
\hline 47 & 4.10 & 33.00 & 3.00 & 765.63 \\
\hline 45 & 4.00 & 48.00 & 3.00 & 785.70 \\
\hline 45 & 4.00 & 52.00 & 3.00 & 785.70 \\
\hline 46 & 2.60 & 48.00 & 3.00 & 416.67 \\
\hline 46 & 3.60 & 52.00 & 3.00 & 612.90 \\
\hline 46 & 2.80 & 50.00 & 3.00 & 416.67 \\
\hline 46 & 2.70 & 28.00 & 3.00 & 416.67 \\
\hline
\end{tabular}

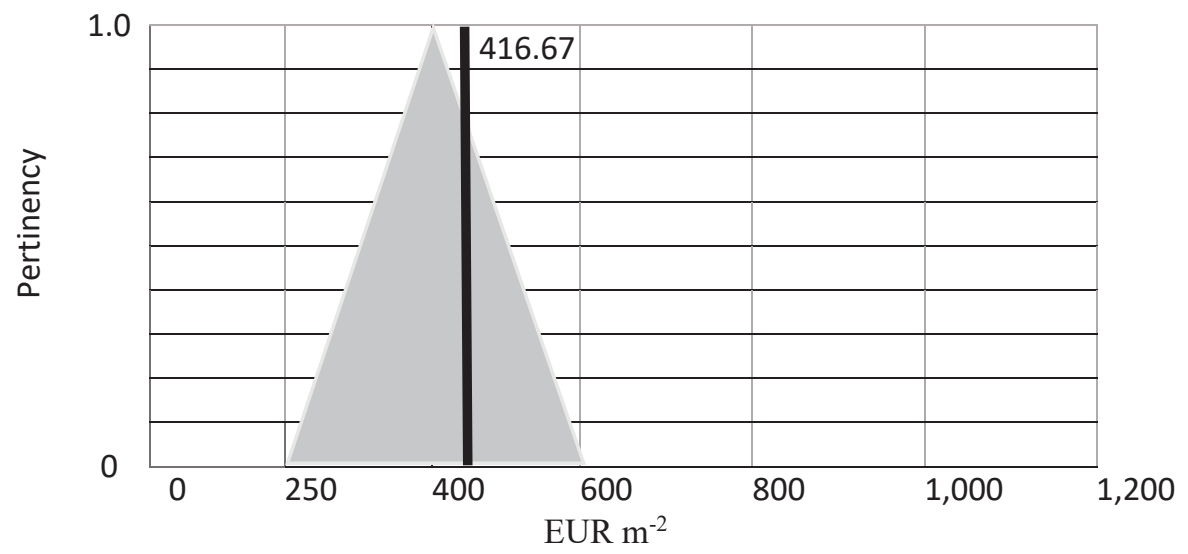

Figure 2. Result obtained by the center of mass criterion (defuzzification method using the infuzzy software). 
Step 4

This experiment performs the comparison between the market values of the property samples with the predicted values from fuzzy logic. The results obtained with the application of the error metrics to evaluate the predictions with the MAPE is $9 \%$. This $9 \%$ result can be interpreted as a good result for this first experiment carried out in the city of Jelgava in Latvia.

\section{Conclusion}

The methodology developed for the evaluation of real estate, using a standardized form of inspection, involving knowledge of civil engineering and artificial intelligence, and fuzzy logic proved to be efficient in this first study. This is because the parameters used in the inspection form proved to be adequate for the reality found in the city of Jelgava, in Latvia. Besides, the fact that it can carry the results of the civil engineering inspection into a heuristic language makes the property assessment process clearer.

Therefore, this experiment contemplates a practical model of property evaluation using a civil engineering inspection form associated with artificial intelligence and diffuse logic and after comparing the market value of these properties, the applicability of this inspection form was verified.

For this, the forecast evaluation follows according to $\mathrm{MAPE}=9 \%$. This means that the difference between the market values and the theoretical value obtained by the diffuse method is reasonable and is within the normal range for this academic experiment. Thus, the comparison of the values obtained in this study with the market values, according to MAPE, represents the possibility of using the methodology developed in this experiment to evaluate properties.

\section{References}

Brandalise, N., Pereira, A. S. A. \& Mello, L. C. B. B. (2019). Aid application multicriteria the decision based on AHP Method and Fuzzy Logic in commercial land selection. Gestão \& Produção, 26(3), e3243. DOI: 10.1590/0104-530X3243-19

Butler, R.V. (1982). Land Economics. Vol. 58, No. 1, pp. 96-108. DOI: $10.2307 / 3146079$
Darlington, M, Zhao, Y, Tang, L, Austin, S \& Culley, S. (2019). Measuring Information Value: key information properties and approaches to assessment. University of Bath, Bath, UK

Del Giudice, V.; De Paola, P.; Cantisani, G.B. (2017). Valuation of Real Estate Investments through Fuzzy Logic. Buildings 2017, 7, 26. DOI:10.3390/buildings7010026

Eibe F., Mark A. H, Ian H. W. (2016). The WEKA Workbench. Online Appendix for "Data Mining: Practical Machine Learning Tools and Techniques", Morgan Kaufmann, Fourth Edition, 2016.

Karaboga, D. \& Kaya, E. (2019). Artif Intell Rev, 52: 2263. DOI: $10.1007 / \mathrm{s} 10462-017-9610-2$

Kusan H., Aytekin O. \& Özdemir I. (2010). The use of fuzzy logic in predicting house selling price. Eskisehir Osmangazi University, Department of Civil Engineering, Turkey. DOI: 10.1016/j. eswa.2009.07.031

Lima, A. J. M. (2013). Engenharia de Avaliações. Universidade Tecnológica Federal do Paraná (UTFPR) (Evaluation Engineering. Federal Technological University of Paraná), Curitiba. (in Portuguese)

MAEC (2006). Método de avaliação do estado de conservação de edifícios. Instruções de aplicação e ficha de avaliação. Ministério Obras Públicas, Transportes e Comunicações (Method of assessing the state of conservation of buildings. Application instructions and evaluation form. Ministry of Public Works, Transport and Communications), Outubro de 2006. LNEC. Lisboa. (in Portuguese)

Posselt, E., Frozza, R. \& Molz, R. (2015). INFUZZY: Ferramenta para desenvolvimento de aplicações de sistemas difusos. Revista Brasileira de computação aplicada (Tool for developing diffuse systems applications. Brazilian Journal of Applied Computin), 7, 1 (fev.2015), 42-52. DOI: 10.5335/rbca.2015.3960. (in Portuguese)

Veiga, M.M. (1994). A heuristic system for environmental risk assessment of Mercury from gold mining operations. DOI: 10.14288/1.0081112

Zadeh, L. A. (1999). Fuzzy sets as a basis for a theory of possibility. Fuzzy Sets and Systems. DOI: 10.1016/0165-0114(78)90029-5.

Research is supported by the Latvian State Scholarship.

\section{Acknowledgments}

